in Polynesia, New Guinea, and elsewhere, have a definite history and meaning yet to be traced. Modern European patterns have also interesting histories to disclose. One of the series in the collection explains the origin of the lozenge- and leaf-pattern common on oak carvings from the intersections of the Gothic arch and ogee arch.

Of the development of ornament from chance marks upon objects, the most interesting example exhibited is probably an Australian boomerang, which happens to have three small round black knots on one side of it placed at equal intervals. The savage owner, struck by the appearance of the knots, burnt a series of similar black marks at equal distances all along the one face of the boomerang, to complete the natural pattern, and then, pleased with his work, put a series of lozenge-shaped marks to correspond on the other side of the weapon. At the Sandwich Islands a most beautiful ornament of the gourds used for water is derived from the net-bag in which the gourds were slung. No doubt the pattern at first became accidentally printed on the gourds, and were afterwards elaborated.

The last series to which we shall draw attention relates to the transformations of the curious ornament which General Pitt Rivers calls the double-loop coil, and which is characteristic of all New Zealand weapons and implements, canoes and houses. The ornament was probably originally copied from coils of string or wire. The distribution of the ornament is very interesting. It is found abundantly in New Guinea, so exactly corresponding to the New Zealand form that it seems certainly to point to some connection between the islanders or partial migration from New Guinea to New Zealand at some time or other, unless some floating object may have conveyed the pattern. A similar ornament occurs in the far-off Marquesas Islands, the natives of which in several other matters of culture show affinity with the Melanesians. It is also very common on Mexican and Peruvian works of art, and especially on gold figures, where it is represented in its former live form by spiral coils of fine wire. From the double-loop coil, as General Pitt Rivers has shown, many other patterns are derived. The fret or key pattern is merely a continuous loop-coil squared. Other patterns, such as the wave pattern, are derived from the coil by slight degeneration. Some of the most marked patterns derived from it are shown in the accompanying figure (Plate 4), which explains itself. It is most curious how nearly parallel the series of modifications attained in the Old and the New World run to one another.

In conclusion we can only express a hope that the Pitt Rivers collection will be accepted by the nation on its generous donor's conditions, and we strongly recommend any of our readers who have not studied it to pay it a long visit at once, and profit by the varied fund of instruction and entertainment which it cannot fail to impart.

\section{THE MASON COLLEGE, BIRMINGHAM}

THE Josiah Mason Science College, which is to be opened by an interesting address from Prof. Huxley to-morrow, was begun about five years ago by the venerable and generous donor, Sir Josiah Mason. It is intended to cover ground not occupied by any other of the numerous educational institutions of Birmingham, to which it promises to be an addition of the highest value. The building itself is described as a lofty and spacious Gothic pile, covering about an acre in extent in the very heart of Birmingham.

By its foundation deed the College is established to provide instruction, as far as possible, in mathematics, abstract and applied; physics, both mathematical and experimental; chemistry, theoretical, practical, and applied; the natural sciences, especially geology and mineralogy, with their application to mines and metal- lurgy; botany and zoology, with special application to manufactures; physiology, with special reference to the laws of health; the English, French, and German languages; and the scheme may, in the discretion of the trustees, include all such other branches of instruction as will conduce to a sound practical knowledge of scientific subjects, excluding mere literary education. The trustees have also power to make provision for instruction in art as well as in science; and, by a supplemental deed, they are authorised to include in the course of study certain subjects requisite for the training of medical students. There is no restriction of the advantages of the college as to sex, creed, or birthplace; but, other things being equal, preference is to be given to candidates who have been educated in Sir Josiah Mason's Orphanage at Erdington, and after these to persons born in Birmingham or Kidderminster, the latter being the founder's birthplace. One wise provision of the deed empowers the trustees, with certain reservations, to alter the course of teaching and the arrangements of the instruction when a change is considered desirable, and at stated intervals the trustees are required to take the arrangements into consideration with a view to revision. At present the branches for which provision is made are confined to mathematics, physics, chemistry, and biology. The mathematical professor is Mr. J. M. Hill, M.A., London, B A., Cantab, Fellow of University College, London. Physics are taught by Prof. J. H. Poynting, M.A., B.Sc., London, Fellow of Trinity College, Cambridge. The chemistry professor is Mr. W. A. Tilden, D.Sc., London, F.R.S.; and biology is represented by Prof. T. W. Bridge, M.A., F.Z.S. According to present arrangements instruction is provided in the elementary as well as the higher branches of the sciences taught, with a special view to their application to the industries of the Midland district. The course is also designed to prepare students for the degrees of B.Sc. and D.Sc. in the University of London.

The internal arrangements seem to be altogether admirable. The main corridor abuts on two noble apartments, each 48 feet by 30 feet - one intended for the library and reading-room, the other for the physical laboratory-both rooms being provided with ante-rooms. On the first floor, the chief and central room, situated in the front of the building, is the chemical lecture theatre, 50 feet by 33 feet, fitted with seats, tier above tier, for the accommodation of 155 students. The male students will occupy the lower half and the female students the seats above and behind them, a separate entrance being provided for each sex. The mechanical arrangements and apparatus for the use of the lecturer and the carrying away of noxious fumes are of the most complete and ingenious character, and the assistants' ante-room, for the preparation of chemical experiments, is on an equally satisfactory scale. Class-rooms for electricity, magnetism, biology, physics, and models, and a couple of spacious lecture theatres, each 47 feet by 30 feet, one for biology and mathematics, the other for physics, occupy the remaining space on the first floor. The second floor is devoted principally to the chemical departments, for which the arrangements are of the most complete and elaborate character. A large room, 52 feet by 33 feet, in the front of the building, over the chemical lecture theatre, will be used as a general assembly or examination room, and will be available for the meetings of scientific societies. The two laboratories situated at the back of the building, and lit both by windows and skylights, measure together about 104 feet long by 32 feet wide. In the larger laboratory, intended for qualitative analysis, there are four double operating-tables fitted with sinks, gas and water for forty students, in addition to a large unencumbered table in the middle of the apartment for long trains of chemical apparatus. The laboratory for quantitative analysis contains similar fittings and appliances 
for thirty-two students. On the third floor a large and lofty central room, with open limber roof, partially lit from the roof, is intended for a museum. The basement story, extending under the whole of the ground floor, is lofty and well lighted, and contains store-rooms, rooms for special operations in physics and chemistry, a large room for mineralogy, rooms for living animals, boilers, \&c. Altogether the building contains at present about 100 rooms. The heating and ventilating arrangements are upon a somewhat novel plan. Near the centre of the area rises a huge chimney-stack to the height of 160 feet, containing three flues divided by thin partitions. The smoke from the boiler passes off by the central flue and heats the air in the adjoining flues, which are used for ventilating the lecture theatres. The warming is effected by a coil of pipes containing 4,475 superficial feet, and fed with water from the large boiler in a vault in the sub-basement. The arrangements in fact are throughout of the most modern and approved types, having been adopted by the architect after mature consideration of all the best features of the principal scientific colleges in this country and on the Continent, which he visited at the request of the trustees.

The generous founder, who has taken a most active interest in the progress of the work, has built the college and furnished its various departments entirely at his own cost, so that the large endowments previously conveyed to the trustees remain untouched. Sir Josiah Mason has stated that his ambition was to afford all classes in the Midland district, where he had been born and bred, the means of carrying on those scientific studies of which he had felt the want as completely and thoroughly as they can be prosecuted in any of the great science schools of Europe.

We earnestly trust that the noble and benevolent intention of the founder will continue to be carried out, and that in time the institution will become as important and comprehensive a centre of higher education as Owens College is now.

\section{THE PROPOSED LICK OBSERVATORY}

M R. S. W. BURNHAM has printed his Report to the I Trustees of the "James Lick Trust" of observations made on Mount Hamilton, California, with reference to the location of the observatory, for the erection and endowment of which funds are thereby provided. His object being to test the adaptation of the site for astronomical purposes by observations of double-stars inainly, Mr. Burnham took with him his 6-inch refractor, by Alvan Clark and Sons, which he has used in nearly all his astronomical work, and the excellence of which has been sufficiently proved by the number of difficult doublestars discovered with it during the last six or eight years. He remained on Mount Hamilton from August I7 to October 16 , and in this interval was in the observatory on every clear night, with three exceptions. During the first thirty-seven nights he states vision was first-class on all occasions with these exceptions; on two nights the ocean fogs from the valley below reached the summit of the mountain and remained all night, and on two other nights there was only medium steadiness. The kind of weather for astronomical observations during the whole period of sixty days that Mr. Burnham remained at the summit, was forty-two first-class nights, seven medium nights, and eleven cloudy and foggy ones. In the whole interval there was not a single poor night when it was clear. By first-class seeing Mr. Burnham explains that he means "such a night as will allow of the use of the highest powers to advantage, giving sharp, well-defined images, and where the closest and most difficult double-stars within the grasp of the instrument can be satisfactorily measured." The conditions were generally very permanent for the whole night, which is not often the case in ordinary localities. On many nights $\mathrm{Mr}$. Burnham remained at the telescope until daylight, and so had abundant opportunities of noting this important fact.

Having provided himself with a series of cardboard disks, with apertures increasing from one inch up to the full aperture of the object-glass, Mr. Burnham observed a large number of familiar objects, contracting the light until the smaller star was just distinctly visible; many of these objects had been used elsewhere for a similar purpose. He considers some of the observations are remarkable, allowing for the difficulty of the objects with much larger apertures in other localities: $\mu^{2}$ Herculis (the close pair) was very fairly seen with the full aperture, and the companion of $a^{2}$ Capricorni was plain with the aperture contracted to 4 inches, and was seen double with the whole six; these objects Mr. Burnham says he is confident have "never been seen before with so small an object-glass." The fifth and sixth stars of $\theta^{2}$ Orionis were very plain at an hour-angle of $4 \frac{1}{2}$ hours ; $\zeta$ Herculiwas well seen with $3 \frac{1}{2}$ in. ; and $\eta$ Cassiopeæ was easy when the aperture was reduced to $\mathrm{I} \frac{1}{2}$ inch. Forty-two new double stars were detected, and micrometrical measures of ninety of these objects previously named were put upon record. A great many were examined by daylight, but the air, during the greater part of the day at least, was not found to be steadier than is ordinarily the case elsewhere. It is mentioned, however, that the fifth and sixth stars of the trapezium of Orion were beautifully seen in broad daylight just before sunrise. At the epoch $1879^{\circ} 684$ the first measure was made fifteen minutes before sunrise, and "both stars were readily seen for some time after this." Venus was very readily seen with the naked eye at any hour of the day, and easily found without any instrumental indication of its place. Mr. Burnham urges that the new double stars brought to light evidence better than anything else can, what may be done at Mount Hamilton, and remarking that these discoveries were effected with an instrument which in these days of great refractors would be regarded as a comparatively inferior telescope, he considers that it is impossible to overestimate the great discoveries which might be made at this station with a first-class object-glass, such for instance as the Naval Observatory, Washington, already possesses, or the proposed Pulkowa glass of twenty-five times the light-power of the one employed; and according to the terms of the Trust the telescope for Mount Hamilton is required to be "superior to and more powerful than any telescope ever yet made;" a condition, however, which perhaps may not be so easily fulfilled as laid down. Mr. Burnham concludes from his experiences on Mount Hamilton that it "offers advantages superior to those found at any point where a permanent observatory has been established." The station is about fifty miles south of San Francisco and twenty-six miles nearly east of San José, the nearest point of railway connection. The ocean fogs, which might have been feared, were not found to reach the elevation, except rarely. Nearly every night this fog, commencing soon after sunset, comes in from the Pacific between the Golden Gate on the north and the Bay of Monterey on the south, and covers the whole valley, but is ordinarily perhaps 2,000 feet below the summit of the mountain, which has an elevation of 4,250 feet above the level of the sea, and has no sensible effect at such altitude.

It will be seen that Mr. Burnham's knowledge of the locality is confined to the space of two months, but a letter from Prof. Davidson of the U.S. Coast Survey, who has had long experience at other seasons, is appended to the report, which is of a very favourable nature, and Mr. Burnham appears to have no hesitation in advising the adoption of Mount Hamilton as the site of the Lick Observatory, which we may hope will be successful in procuring an instrument worthy of the other great astronomical 